Note: this is a post-print draft of the journal article:

2

3 Dickinson, A.S., Taylor, A.C., Browne, M. (2012) "The Influence of Acetabular Cup Material on Pelvis

4 Cortex Strains, Measured using Digital Image Correlation". Journal of Biomechanics, 45 pp719-723

5

6 The final, fully proofed and peer-reviewed journal article is available from the publisher online, via

7 the following link:

8 http://www.sciencedirect.com/science/article/pii/S002192901100724X\#FCANote

9 http://dx.doi.org/10.1016/j.jbiomech.2011.11.042

10 


\section{Digital Image Correlation}

A.S. Dickinson ${ }^{1,2}$, A.C. Taylor ${ }^{2}$, M. Browne ${ }^{1}$

15

1: University of Southampton, Southampton, UK

2: Aurora Medical Ltd., Chilworth, UK

17

Corresponding Author:

A.S. Dickinson

20

Bioengineering Research Group,

21 School of Engineering Sciences,

22 University of Southampton,

23 Highfield,

24 Southampton,

25 United Kingdom.

26 alex.dickinson@soton.ac.uk

27 Tel: $+44(0) 2380592443$

Fax: $+44(0) 2380593016$

Short Communication: Word Count (Introduction through Discussion) $=2024$ Words

Keywords: Bone Strain Measurement, Stress Shielding, Implant Biomaterials, Hip

32 Replacement

33 All authors have made a substantial contribution to this work, have read and concur with 34 the content of the manuscript.

35 This work has not been submitted for publication elsewhere, but was presented as a poster at the ISTA conference 2011. 
Abstract:

Acetabular cup loosening is a late failure mode of total hip replacements, and peri-prosthetic bone deterioration may promote earlier failure. Preservation of supporting bone quality is a goal for implant design and materials selection, to avoid stress shielding and bone resorption. Advanced polymer composite materials have closer stiffness to bone than metals, ceramics or polymers, and have been hypothesised to promote less adverse bone adaptation. Computer simulations have supported this hypothesis, and the present study aimed to verify this experimentally.

A composite hemi-pelvis was implanted with Cobalt Chromium ( $\mathrm{CoCr}$ ), polyethylene (UHMWPE) and MOTIS ${ }^{\circledast}$ carbon-fibre-reinforced polyether ether ketone (CFR-PEEK) acetabular cups. In each case, load was applied to the implanted pelvis and Digital Image Correlation (DIC) was used for surface strain measurement. The test was repeated for an intact hemi-pelvis. Trends in implanted vs. intact bone principal strains were inspected to assess the average principal strain magnitude change, allowing comparison of the potential bone responses to implantation with the three cups.

The CFR-PEEK cup was observed to produce the closest bone strain to the intact hip in the main load path, the superior peri-acetabular cortex $\left(+12 \%\right.$ on average, $\left.\mathrm{R}^{2}=0.84\right)$, in comparison to $\mathrm{CoCr}$ $\left(+40 \%, R^{2}=0.91\right)$ and UHWMPE cups $\left(-26 \%, R^{2}=0.94\right)$. Clinical observations have indicated that increased periacetabular cortex loading may result in reduced polar cancellous bone loading, leading to longer term losses in periprosthetic bone mineral density. This study provides experimental evidence to verify previous computational studies, indicating that cups produced using materials with stiffness closer to cortical bone recreate physiological cortical bone strains more closely and could, therefore, potentially promote less adverse bone adaptation than stiffer press-fitted implants in current use. 
Aseptic loosening is the most commonly reported indicator for revision of total hip replacements, with acetabular cups revised more commonly than femoral stems $[1,2]$. Notwithstanding possible incorrect cup positioning and wear-induced osteolysis, retrieval evidence suggests that loosening may be linked to increased bearing friction late in the implant's life [3]. Maintenance of supporting bone quality would delay loosening, but reduced bone mineral density (BMD) has been measured in the periprosthetic bone near the pole of press-fit cups [4-10], in a pattern consistent with adaptive remodelling. Periprosthetic bone deterioration may promote earlier failure, so preservation of supporting bone quality is a goal for implant designers.

Excessively stiff implants are thought to alter the strain field in the supporting bone, potentially causing loosening by stress shielding and bone resorption [11-13]. Recent developments in advanced polymer composite technology have produced bearing materials with low long-term wear and closer stiffness $(E)$ to bone tissue $(E \approx 17 G P a)$ than metals ( $\approx \approx 200 G P a)$, ceramics $(E \approx 350 G P a)$ or polymers $(\mathrm{E} \approx 0.9 \mathrm{GPa})$. Accordingly, these materials are predicted to promote less adverse bone adaptation, a theory which has been supported by computer simulations $[12,14,15]$.

It is established that the biomechanical bone adaptation stimulus resulting from implantation can be assessed by measuring the change from pre- to post-operative peri-prosthetic strains. Digital Image Correlation (DIC) is a non-contact, full surface strain measurement technique which has been applied in several biomechanical scenarios [16-18]. In the present study, DIC was used to analyse the change in peri-acetabular strains caused by implantation with cups made from three materials of different stiffness. The aim was to retest the hypothesis that acetabular cups produced from materials with closer stiffness to cortical bone will promote less adverse bone adaptation than high stiffness metal cups, using experimental testing to verify past computational predictions. 
86 A composite hemi-pelvis (\#3405, Sawbone AB, Sweden) was reamed for a 58mm outer87 diameter (OD), 52mm inner-diameter (ID) cobalt chromium ( $\mathrm{CoCr}, \mathrm{E}=197 \mathrm{GPa}$ ) ADEPT 88 acetabular cup (Mat Ortho Ltd., UK) with approximately $0.5 \mathrm{~mm}$ diametric press-fit. The 89 model was mounted on an Instron 8874 servo-hydraulic axial test machine (Instron Corp., 90 USA) using a fixture giving sacroiliac and pubic symphysis support with adjustable 91 abduction-adduction and flexion-extension angles (Fig.1). The model was oriented so that 92 the machine applied a generalised $1500 \mathrm{~N}$ joint contact force, in $12^{\circ}$ adduction. 


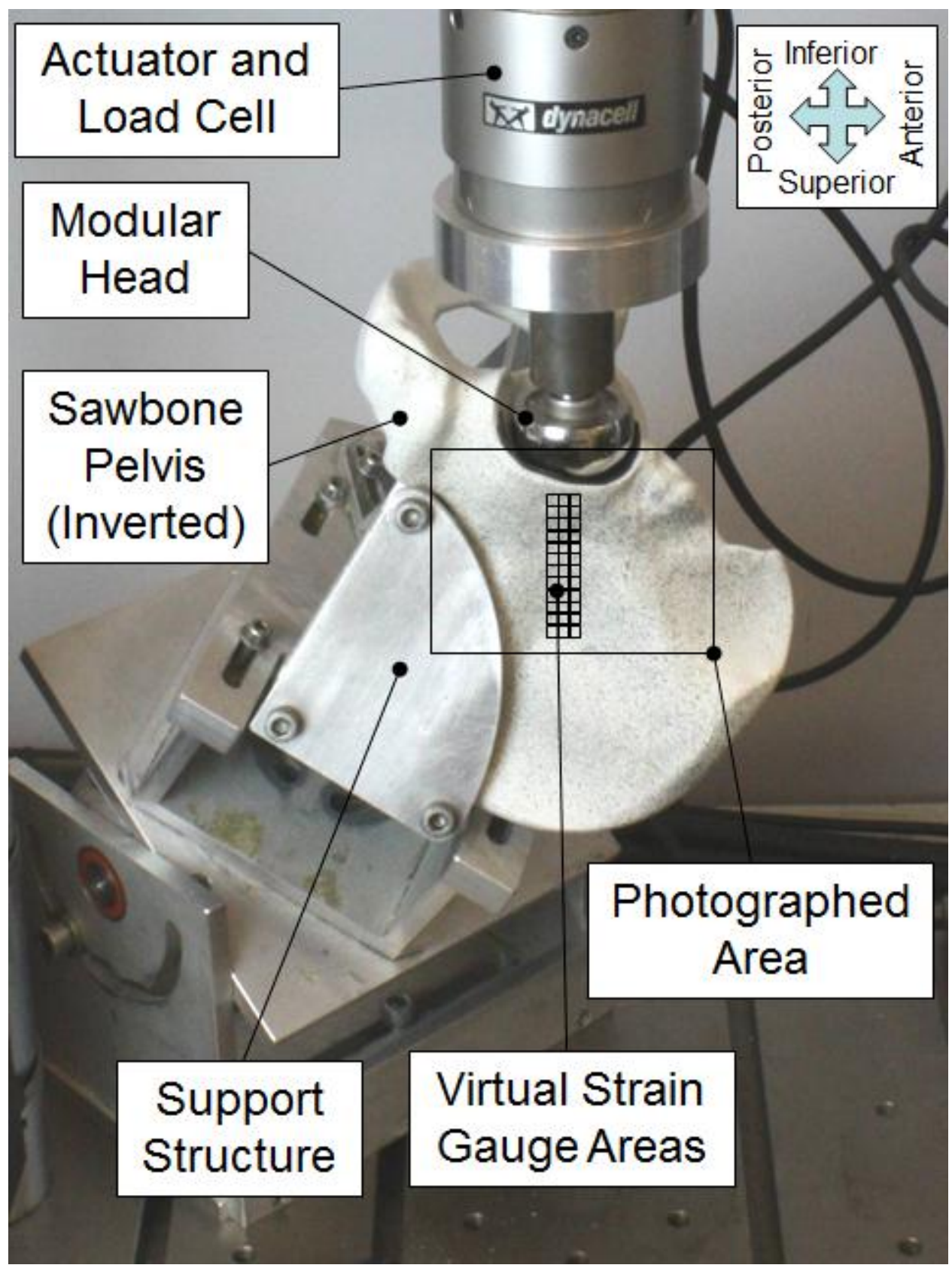

Figure 1 : Mechanical Test Setup (Intact Case Shown).

DIC was used for strain measurement on the cortical bone surface according to a previously verified technique [18]. A speckle pattern was applied to the anterio-lateral bone surface superior to the cup with an airbrush. Locations and displacements of the pattern features were recorded by dual 2MP digital cameras (Limess GmbH, Germany), and VIC3D software (Correlated Solutions Inc., USA) was used to calculate displacements and principal 
101

strains under loading. Five repeat unloaded datasets were collected to assess measurement sensitivity, and five repeat loaded datasets were collected to assess measurement variability. Three other scenarios were then tested:

- implanted with a $52 \mathrm{~mm}$ ID press-fitted MOTIS CFR-PEEK composite cup (carbon-fibrereinforced PEEK, E=12-15GPa, approximately isotropic, with short pitch fibres $\sim 150 \mu \mathrm{m}$ length, 7 $\mu \mathrm{m}$ diameter, Invibio Biomaterial Solutions, UK),

- implanted with a $28 \mathrm{~mm}$ ID cemented UHMWPE polymer cup (ultra-high-molecularweight-polyethylene, E $\approx 0.9 \mathrm{GPa}$ ) using Smartset medium viscosity PMMA bone cement (DePuy CMW, UK), and

- using a second, intact hemi-pelvis to obtain reference strains.

Reproducible implant positioning was ensured in all three implanted cases by using the same reamed bone, and by locating the cup rim relative to two points on the anterior and superior acetabular rim. The $\mathrm{CoCr}$ and CFR-PEEK cups were loaded with a $52 \mathrm{~mm} \mathrm{CoCr}$ ADEPT modular head, and the UHMWPE cup with a $28 \mathrm{~mm}$ BIOLOX forte modular head (CeramTec AG, Germany). The intact bone was loaded with a $48 \mathrm{~mm} \mathrm{CoCr}$ ADEPT modular head and a thin rubber interlayer to encourage uniform load transfer over the acetabular bearing surface. The rubber layer was $3 \mathrm{~mm}$ thick, representing the combined thickness of femoral and acetabular cartilage, and had a compressive modulus of approximately $10 \mathrm{MPa}$, within the range of human cartilage stiffness under physiological loading rates $[19,20]$.

Use of the same reamed bone was also intended to ensure the same implant-bone pressfit for the CoCr and CFR-PEEK cups. To check that peri-acetabular bone yield did not occur between successive implantations, diminishing the press-fit, the peak peri-acetabular stress was measured in each test. This was judged to be valid because the majority of the load 
transfer in the press-fit cups was through the cortical bone, and only focussed regions of cancellous bone on the lunate acetabular surface were uncovered upon reaming. The peak tensile stress of $15.5 \mathrm{MPa}$ represented $13.7 \%$ of the material's tensile strength of $106 \mathrm{MPa}$, and the peak compressive stress of $21.5 \mathrm{MPa}$ represented $14.6 \%$ of the $157 \mathrm{MPa}$ compressive strength [21].

The strain in the implanted bone was averaged across thirty-six $5 \mathrm{~mm} \times 5 \mathrm{~mm}$ gauge areas superior to the acetabular rim, and compared to the intact bone strain for an indication of the remodelling stimulus [22] for all three implant materials. Scatter graphs of implanted vs. intact strain were plotted, trend lines were fitted to the data and the gradients were inspected to assess the average principal strain magnitude change. This allowed quantitative comparison of predicted bone responses to implantation with $\mathrm{CoCr}$, UHMWPE and CFR-PEEK cups.

\section{Results}

Assessing measurement sensitivity, the error in principal strains was $203.7 \mu \varepsilon$ (tension) and $224.4 \mu \varepsilon$ (compression), calculated as the mean plus 3 standard deviations $(99.7 \%$ confidence) in the five unloaded tests.

Principal strain maps for the intact and implanted tests (Fig.2) show a clear load path in the cortex from the acetabulum up to the sacroiliac joint. This is in close agreement with the computational study that employed the most physiologically representative, flexible, musculo-ligamentous boundary conditions [23]. The CoCr and CFR-PEEK cups generated increased tensile and compressive cortex strains superior to the acetabular rim, whereas the UHMWPE cup generated a global reduction in cortex strain. 


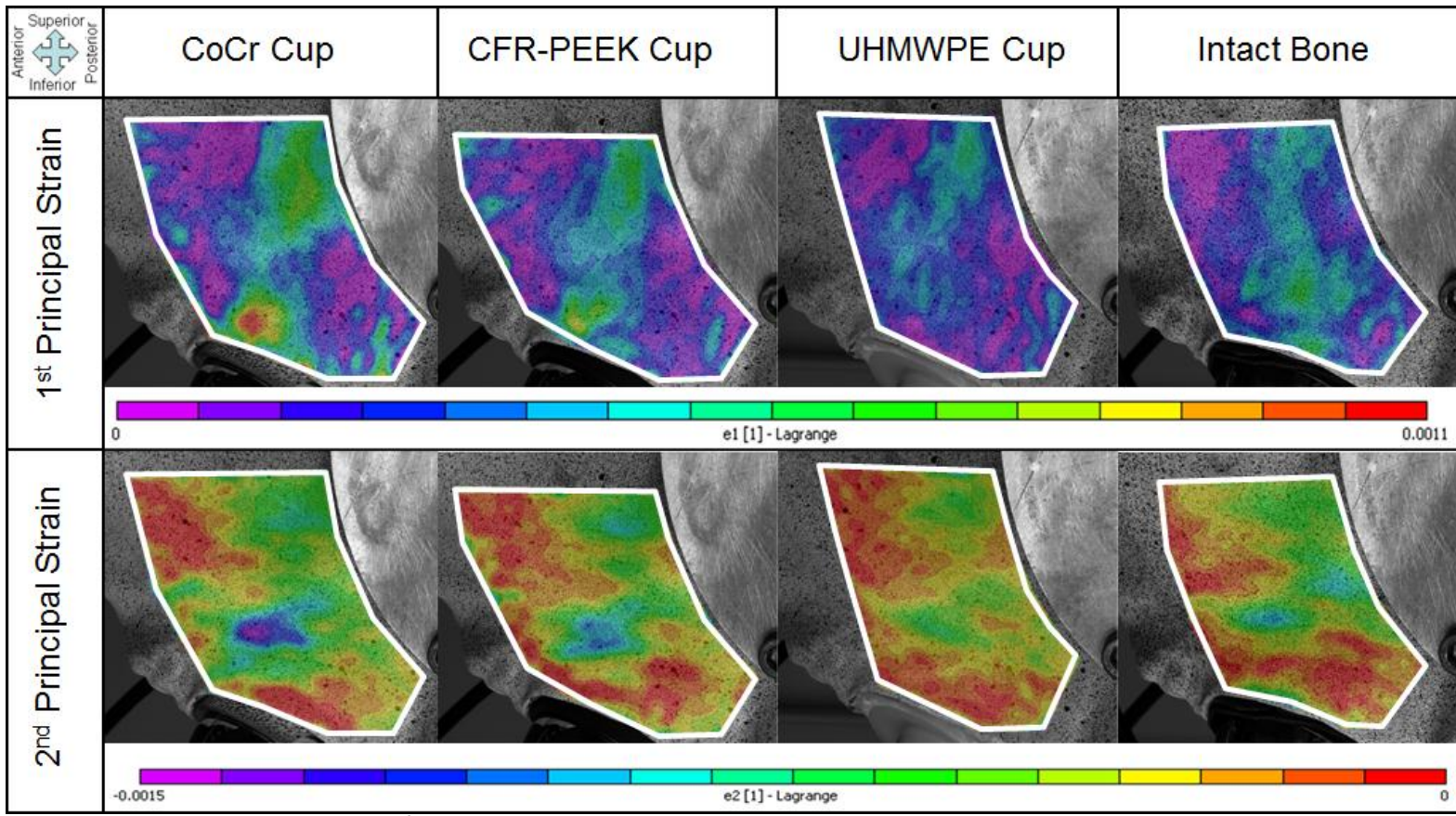

Figure 2: $1^{\text {st }}$ and $2^{\text {nd }}$ Principal Strain Maps for the Intact and three Implanted Tests.

Implanted vs. intact bone strain scatter graphs are presented for the three cups in Fig.3,

151 for quantitative analysis. The average principal strain magnitude in the peri-acetabular

152 cortical bone was increased by $40 \%$ after implantation with the $\operatorname{CoCr}$ cup $\left(R^{2}=0.84\right)$, and

strain magnitude in the gauge region by $12 \%\left(R^{2}=0.91\right)$.

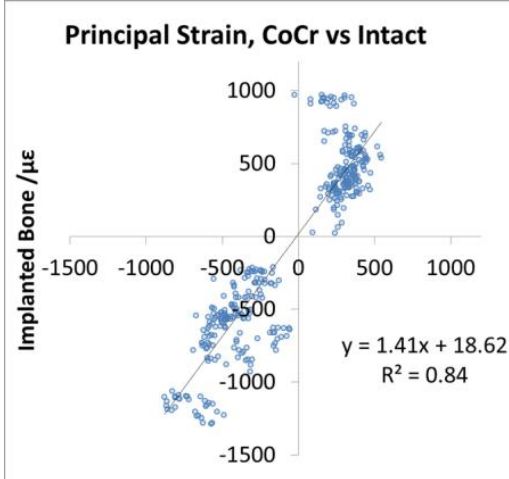

Intact Bone / $\mu \varepsilon$
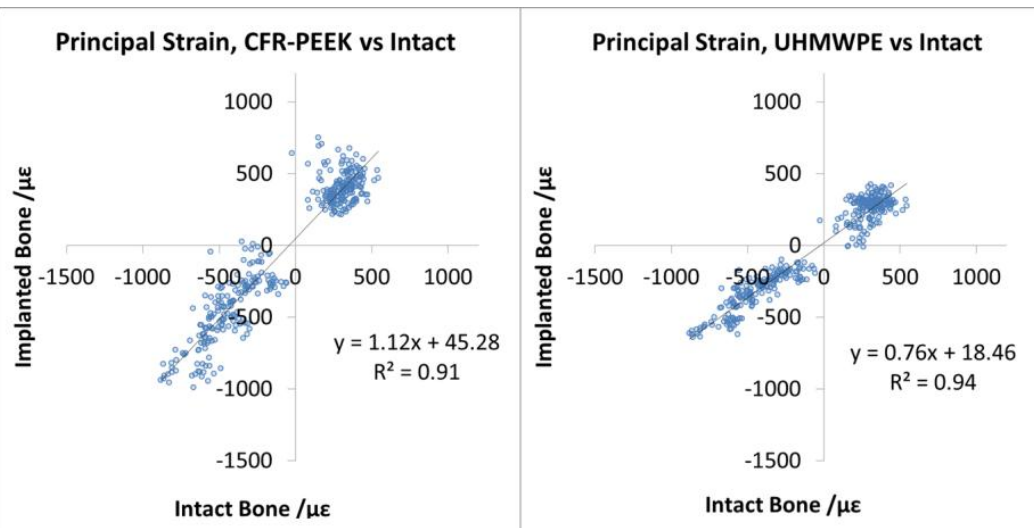

Figure 3: Comparison of Principal Strain in Intact and Implanted Tests 
This study set out to test the hypothesis that implant biomaterials with stiffness similar to cortical bone would reproduce more closely the intact joint's more diffuse bone strain distribution, as has been indicated clinically by porous metallic cups [10]. Clinical radiographic measurements have indicated that contemporary cementless acetabular cups preferentially load the acetabular rim, and shield the central ilium from load $[5,8]$. This has been identified by a significant loss of bone mineral density superior to the pole of cementless cups, which stabilises after the first postoperative year $[4-8,10]$ indicating an adaptive process. The results confirm the hypothesis, with a CFR-PEEK cup generating a smaller average increase in cortex strains, and less acetabular rim cortex strain concentration than a CoCr cup. An UHMWPE cup was also tested, with even lower stiffness, and this produced a global reduction in cortex strain, consistent with its higher flexibility causing reduced rim load transfer to the cortex, and increased polar load transfer to the cancellous bone.

This study's results are corroborated by clinical DEXA scan measurements [5-10], cadaveric implant-bone load distribution measurements [24] and previously published numerical predictions $[14,15]$ which indicated that stiffer metal cups load the superior acetabular rim cortex preferentially, whereas polymeric cups transferred load more evenly. Implant material is not the only factor; clinical evidence has shown that cemented cups produce a more natural load transfer pattern than press-fitted implants [9]. Thompson et al [14] also predicted that interface conditions are more influential upon peri-acetabular bone strain changes, and the present results for the UHMWPE cup will have been influenced by its cemented fixation. However, Thompson et al's predictions indicate that cups with 
bonded interfaces load the cortical bone preferentially to the subchondral bone. Therefore, this study's measured reduction in cortical bone loading and predicted increase in polar cancellous bone loading with the UHMWPE cup is predicted to be conservative compared with the un-bonded fixation of the metal and composite cups.

The results must be interpreted with consideration of their limitations. The strains in the superior-lateral portion of the cortical bone were considered alone, as the DIC technique was not capable of measuring internal strains within the cancellous bone, and because lineof-sight access was not available on the medial cortex surface. The cortex in the superior peri-acetabular region was the focus of this study because it is the main load transfer path to the sacroiliac joint. Therefore, conclusions can only be drawn considering the stimulus for cortical bone adaptation, but predictions of resulting cancellous adaptations may also be made. Clinical observations have informed the suggestion that increased peri-acetabular cortical load transfer may lead in turn to reduced polar cancellous load transfer $[5,8]$, so it can be predicted that an implant which reproduces the intact bone's cortical strain distribution more closely will also produce more physiological cancellous bone strains.

A further limitation is that the study employed an in-vitro model. A single bone model, load case and prosthesis position was used, neglecting muscle forces; this was intended to represent a generalised, approximately clinically representative situation so that a like-forlike comparison could be made. An analogue bone was used instead of cadaver material as it represented a consistent, widely available model designed to behave in a globally similar manner to real bone. A minimal press fit was used for the cementless cups, so the data neglects residual strain. This was justified because it is likely to represent a conservative case, where the stiffer $\mathrm{CoCr}$ cup would theoretically produce greater residual strain than the 
CFR-PEEK cup, and the UHMWPE cup would produce negligible residual strains. Inclusion of residual strains would theoretically strengthen the observed trends, but would also be gradually relieved through viscoelastic effects [25] and bone adaptation. The predicted strain patterns are representative of the short-term postoperative case, but would be influenced by progressive osseointegration and periprosthetic bone adaptation. strain measurement locations differed from the three implanted cases. The effects of measurement variability were minimised by taking repeat measurements, but experimental variability could be quantified in absolute terms by further repeat tests. It is proposed, however, that the single intact case is sufficient for the comparative analysis employed. bone strain is only one factor which influences bone adaptation. Excessive relative micromotion at the implant-bone interface has been shown to lead to the formation of nonmineralised, fibrous tissue which is incapable of supporting the implant $[26,27]$. More flexible prostheses may reduce stress shielding, but excessive flexibility could increase the local interface stress, potentially leading to loosening through micromotion-stimulated fibrous tissue formation [12]. More flexible prostheses would require careful consideration

222 of fixation, which was not included in the present study. Furthermore, predictions by Manley et al [15] indicated that while material selection can improve the load transfer to 224 some extent, modification of the cup design from an axisymmetric hemispherical shell may be necessary to reproduce more natural strain distributions. The observed effects of the cup material may be specific to the design which was tested, so future investigations could 
investigate a range of relevant parameters such as additional cup designs, materials and

228 MB receives studentship research funding from Invibio Ltd for other projects.

fixation methods.

In conclusion, a MOTIS CFR-PEEK composite acetabular cup was tested in an analogue model, and measured to produce the closest bone strain to the intact pelvis in the main load path, the superior peri-acetabular cortex, compared to clinically-used $\mathrm{CoCr}$ metal and UHMWPE polymer cups. In this case, it may be predicted to produce a lower extent of internal cancellous bone stress shielding than stiffer cups in clinical use, supporting the hypothesis. The study underpins the use of DIC for biomechanical assessments of surface strain. The results provide experimental evidence to support computational predictions which indicate that cups produced using materials with stiffness closer to cortical bone may recreate physiological cortical bone strains more closely, potentially inducing less adverse bone adaptation and offering greater longevity.

\section{Acknowledgments}

This study was funded by a European Union $7^{\text {th }}$ Framework programme. The authors would like to thank Invibio Biomaterial Solutions for supplying the CFR-PEEK material, used to produce the test specimens, and Mr Matthew Kelly for conducting preliminary testing.

\section{Conflict of Interest Statement}

None of the Authors has a Conflict of Interest associated with this study.

248 


\section{References}

251 1. National Joint Registry for England and Wales 5th Annual Report. 2008: Hemel Hempstead.

252 2. Australian Orthopaedic Association National Joint Replacement Registry Annual Report. 2009, AOA: Adelaide.

253 3. Tuke, M.A., Scott, G, Roques, A, Hu, X Q, Taylor, A C, Design Considerations and Life Prediction of Metal-on-Metal

254 Bearings: The Effect of Clearance. J Bone Joint Surg [Am], 2008. 90: p. 134-141.

255 4. Sabo, D., Reiter, A, Simank, H G, Thomsen, M, Lukoschek, M, Ewerbeck, V, Periprosthetic Mineralization Around Cementless Total Hip Endoprosthesis: Longitudinal Study and Cross-Sectional Study on Titanium Threaded Acetabular Cup and Cementless Spotorno Stem with DEXA. Calcif Tissue Int, 1998. 62: p. 177-182.

5. Wright, J.M., Pellicci, P M, Salvati, E A, Gehlman, B, Roberts, M M, Koh J L, Bone Density Adjacent to Press-Fit Acetabular Components: a Prospective Analysis with Quantitative Computed tomography. J Bone Joint Surg [Am], 2001. 83-A: p. 529-536.

6. Stolk, J., Dormans, K W, Sluimer, J, van Rietbergen, B, Geesink, R G, Huiskes, R. Is Early Bone Resorption around Non-Cemented THA Cups Related to Stress Shielding? in 50th Annual Meeting of the Orthopaedic Research Society. 2004. San Francisco.

7. Laursen, M.B., Nielsel, P T, Søballe, K, Bone Remodelling around HA-Coated Acetabular Cups: A DEXA Study with a 3-Year Follow-Up in a Randomised Trial. Int Orth, 2007. 31: p. 199-204.

8. Pitto, R.P., Bhargava, A, Pandit, S, Munro, J T, Retroacetabular Stress Shielding in THA. Clin Orth Rel Res, 2008. 466: p. 353-358.

9. Mueller, L.A., Schmidt, R, Ehrmann, C, Eckhard Nowak, T, Kress, A, Forst, R, Pfander, D, Modes of Periacetabular Load Transfer to Cortical and Cancellous Bone after Cemented versus Uncemented Total Hip Arthroplasty: A Prospective Study using Computed Tomography-Assisted Osteodensitometry. J Orth Res, 2009. 27: p. 176-182.

10. Meneghini, R.M., Ford, K S, McCollough, C H, Hanssen, A D, Lewallen, D G, Bone Remodelling around Porous Metal Cementless Acetabular Components. J Arthoplasty, 2010. 25: p. 741-747.

11. Lewis, J.L., Askew, M J, Wixson, R L, Kramer, G M, Tarr, R R, The Influence of Prosthetic Stem Stiffness and of a Calcar Collar on Stresses in the Proximal End of the Femur with a Cemented Femoral Component. J Bone Joint Surg, 1984. 66-A: p. 280-286.

12. Huiskes, R., Weinans, M S, van Rietbergen, M S, The Relationship between Stress Shielding and Bone Resorption around Total Hip Stems and the Effects of Flexible Materials. Clin Ortho Rel Res, 1992. 274: p. 124-134.

13. Bobyn, J.D., Mortimer, E S, Glassman, A H, Engh, C A, Miller, J E, Brooks, C E, Producing and avoiding stress shielding: laboratory and clinical observations of noncemented total hip arthroplasty. Clin Ortho Rel Res, 1992. 274: p. 79-96.

14. Thompson, M.S., Northmore-Ball, M D, Tanner, K E, Effects of acetabular resurfacing component material and fixation on the strain distribution in the pelvis. Proc IMechE H, 2002. 216: p. 237-245.

15. Manley, M.T., Ong, K L, Kurtz, S M, The Potential for Bone Loss in Acetabular Structures Following THA. Clin Orth Rel Res, 2006. 453: p. 246-253.

16. Thompson, M.S., Schell, H, Lienau, J, Duda, G N, Digital Image Correlation: A Technique for Determining Local Mechanical Conditions within Early Bone Callus. Med Eng and Physics, 2007. 29: p. 820-823.

17. Sztefek, P., Vanleene, M, Olsson, R, Collinson, R, Pitsillides, A A, Shefelbine, S, Using Digital Image Correlation to Determine Bone Surface Strains During Loading and After Adaptation of the Mouse Tibia. J Biomech, 2010. 43: $\mathrm{p}$. 599-605.

18. Dickinson, A.S., Taylor, A C, Ozturk, H, Browne, M, Experimental Validation of a Finite Element Analysis Model of the Proximal Femur using Digital Image Correlation and a Composite Bone Model. Journal of Biomechanical Engineering, 2011. 133.

19. Shepherd, D.E.T., Seedhorn, B B, A Technique for Measuring the Compressive Modulus of Articular Cartilage under Physiological Loading Rates with Preliminary Results. Proc IMechE Part H: J Eng Med, 1997. 211: p. 155165.

20. Shepherd, D.E.T., Seedhorn, B B, Thickness of Human Articular Cartilage in Joints of the Lower Limb. Ann Rheum Dis, 1999. 58: p. 27-34.

21. MatWeb Website, www.matweb.com. Material Property Data. October 2011].

22. Taylor, M., Finite element analysis of the resurfaced femoral head. Proc IMechE H, 2006. 220: p. 289-297.

23. Phillips, A.T.M., Pankaj, P, Howie, C R, Usmani, A S, Simpson, A H R W, Finite element modelling of the pelvis: Inclusion of muscular and ligamentous boundary conditions. Med Eng Phys, 2007. 29: p. 739-748.

24. Widmer, K.-H., Zurfluh, B, Morscher, E W, Load Transfer and Fixation Mode of Press-Fit Acetabular Sockets. J Arthroplasty, 2002. 17: p. 926-935.

25. Cotton, J.R., Zioupos, P, Winwood, K, Taylor, M, Analysis of Creep Strain during Tensile Fatigue of Cortical Bone. J Biomech, 2003. 36: p. 943-949.

26. Pilliar, R.M., Lee, J M, Maniatopoulos, C D D S, Observations on the Effect of Movement on Bone Ingrowth into Porous-Surfaced Implants. Clin Orth, 1986. 208: p. 108-113.

27. Søballe, K., Hydroxyapatite ceramic coating for bone-implant fixation. Mechanical and histological studies in dogs. Acta Orthop Scand, 1993. 65 S255: p. 1-58. 